\title{
A Study on the Scattering Energy Properties of an Elastic Spherical Shell in Sandy Sediment Using an Improved Energy Method
}

\author{
WenKai Wang, GuangPing Zhu, Hui Sun, JingWei Yin, and MingHui Zhang \\ College of Underwater Acoustic Engineering, Harbin Engineering University, Harbin 150001, China \\ Correspondence should be addressed to GuangPing Zhu; e5-2670@outlook.com
}

Received 23 September 2016; Accepted 22 December 2016; Published 13 February 2017

Academic Editor: Andrea Crivellini

Copyright (c) 2017 WenKai Wang et al. This is an open access article distributed under the Creative Commons Attribution License, which permits unrestricted use, distribution, and reproduction in any medium, provided the original work is properly cited.

An elastic wave is composed of compressional (longitudinal) waves and shear (transverse) waves which have different wave velocities in solids. The acoustic field presents complex interference patterns which means its phenomena and properties are difficult to reveal. Fortunately, the energy method is more accurate than the potential function approach in describing the physical properties of the acoustic field. However, the polarization state of particle vibration excited by an elastic wave is spatially periodic in the wave propagation direction. Therefore, the energy propagation direction is not consistent with the wave propagation direction using commonly used energy method. According to the polarization state of particle vibration, a time-space averaging method based on the spatial periodicity of energy flux in the solid is proposed. The method could eliminate the influence of the interference due to local energy exchange and retain the trend of energy propagation. Several conclusions are illustrated through the analysis of the scattering energy properties of a steel shell in sandy sediment. Sandy sediment can not be regarded as a fluid nor a general solid. Scattering energy excited by an incident shear wave mainly concentrates in the vicinity of the directions of backscattering and forward scattering. Especially, at low frequency, it plays an important role in the total scattering energy excited by an incident compressional and shear wave.

\section{Introduction}

The acoustic field in solids presents a complex interference pattern because the compressional wave and the shear wave have different propagation velocities which both have effects on particle velocity, although the usual energy method is more accurate than the potential function approach in describing the physical properties of acoustic fields. As yet no such energy method is applicable to describe the energy propagation in solids because the energy propagation direction is not consistent with the wave propagation direction using usual energy methods.

The problem of the scattering field in solids has been studied in detail by scholars [1-3]. But there is rare research on the acoustic energy propagation. The energy density and energy flux vectors derived with the aid of Hamilton's principle and Umov-Poynting's theorem is obtained by Pierce [4].
The time-average energy density is obtained from the projection of the average power flow vector onto the propagation direction by Carcione and Cavallini [5]. The further study found that the relevant physical phenomena are related to the energy flow direction rather than to the propagation direction [6]. Time averaged Umov-Poynting vector is defined as acoustic intensity by Ainslie and Burns [7] when discussing the energy of reflected wave and transmitted wave at the interface of solid. An algorithm is used for calculating Umov-Poynting vector and the pressure force exerted by the nonparaxial cylindrical Gaussian wave on a circular microcylinder by Kotlyar et al. [8]. The acoustic power radiated by plates in bending vibration is estimated using Statistical Energy Analysis by Rumerman [9]. The instantaneous Umov-Poynting vector field is shown in both freeze-frame and animated versions in Dean and Braselton's research [10-12]. The Umov-Poynting vector is applied to 


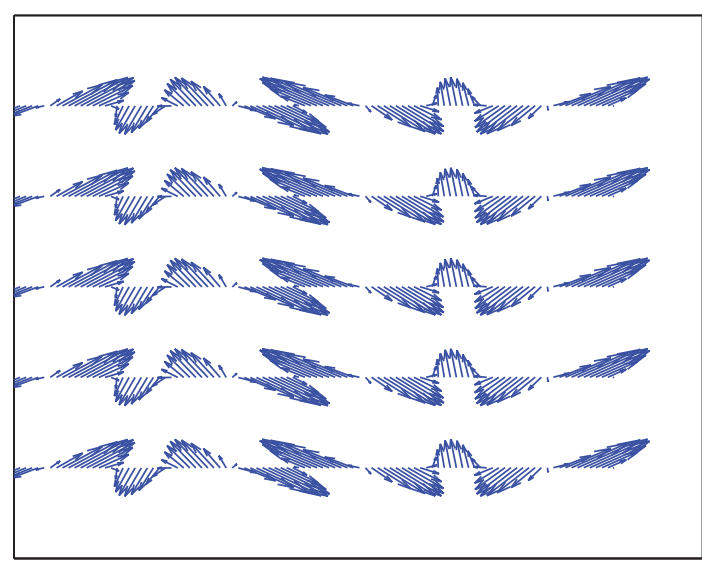

(a)

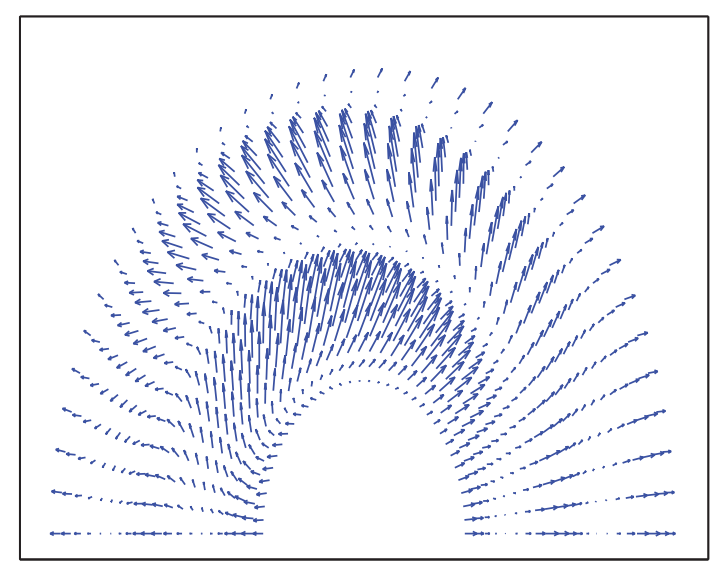

(b)

FIgURe 1: Acoustic Umov-Poynting vector. (a) Incident wave; (b) scattered wave.

solve problems of thermal conductivity in any media specifying the medium and values of heat fluxes at the interface by Kuts [13]. The statements of the elasticity problem about the wave propagation in an elastic cylindrical waveguide under different radiation conditions at infinity are considered by Nazarov [14]. The sound radiation from elastically restrained plates covered by a decoupling layer is studied using the Spectrogeometric Method by Wang et al. [15].

Present researches are focusing on the time average of the instantaneous energy flux. But, the physical image of energy propagation is too complex to understand this way. This paper presents a time-space average method by which the physical image and the properties of acoustic energy propagation in the solid are accurately described. The elastic scattering energy of a spherical shell in sandy sediment especially is analyzed using this method.

\section{Instantaneous Scattering Energy Flux of Elastic Spherical Shell}

Each of the physical quantities involved in the acoustic energy flux are derived from potential functions. The vector Helmholtz equation $\nabla^{2} \mathbf{s}+k^{2} \mathbf{s}=0$ is introduced in order to obtain the analytical solution of the scattered acoustic field, where $\mathbf{s}$ is the displacement vector in solids and it can be decomposed into the following form by field theory:

$$
\mathbf{s}=\mathbf{L}+\mathbf{M}+\mathbf{N},
$$

where $\mathbf{L}, \mathbf{M}$, and $\mathbf{N}$ satisfy the vector Helmholtz equation. The vector solutions are related to the scalar functions as follows:

$$
\begin{aligned}
\mathbf{L} & =\frac{\nabla \phi}{k}, \\
\mathbf{M} & =\nabla \times\left(\mathbf{e}_{r} r \psi\right), \\
\mathbf{N} & =\frac{\nabla \times \nabla \times\left(\mathbf{e}_{r} r \chi\right)}{k},
\end{aligned}
$$

where $\phi, \psi, \chi$ satisfy the scalar Helmholtz equation.
The scattering displacement vector is given by

$$
\begin{aligned}
\mathbf{u}_{s}(\mathbf{r}) & =\sum_{m} \sum_{n} i^{n} \frac{2 n+1}{n(n+1)}\left[a_{m n} \mathbf{M}_{o m n}^{3}(\mathbf{r})-i b_{m n} \mathbf{N}_{e m n}^{3}(\mathbf{r})\right. \\
& \left.+d_{m n} \mathbf{L}_{e m n}^{3}(\mathbf{r})\right] .
\end{aligned}
$$

The undetermined coefficients are obtained using the boundary conditions for displacement and stress. The simplified scattering displacement in the far field is derived using an approximation for the Hankel function:

$$
\begin{aligned}
& u_{s r}(r, \theta, \phi)=\sum_{m} \sum_{n} \frac{2 n+1}{n(n+1)} \frac{e^{i k r}}{k r} d_{m n} P_{n}^{m}(\cos \theta) \\
& \cdot \cos m \phi, \\
& u_{s \theta}(r, \theta, \phi)=\sum_{m} \sum_{n} \frac{2 n+1}{n(n+1)} \frac{e^{i K r}}{i K r}\left[a_{m n} \frac{P_{n}^{m}(\cos \theta)}{\sin \theta}\right. \\
& \left.\quad+b_{m n} \frac{\mathrm{d} P_{n}^{m}(\cos \theta)}{\mathrm{d} \theta}\right] \cos m \phi, \\
& u_{s \phi}(r, \theta, \phi)=\sum_{m} \sum_{n} \frac{2 n+1}{n(n+1)} \frac{i e^{i K r}}{K r}\left[a_{m n} \frac{\mathrm{d} P_{n}^{m}(\cos \theta)}{\mathrm{d} \theta}\right. \\
& \left.\quad+b_{m n} \frac{P_{n}^{m}(\cos \theta)}{\sin \theta}\right] \sin m \phi,
\end{aligned}
$$

where $k$ is the compressional wavenumber while $K$ is the shear wavenumber. From expressions (4)-(6), the radial displacement is the compressional component of the scattered wave, while the tangential and circumferential displacement is the shear component of the scattered wave. The scattered field has three displacement components and six stress components. It is difficult to reveal more scattering rules even if the expressions are very succinct. In this paper we introduce the acoustic Umov-Poynting vector:

$$
\mathbf{P}=-\mathbf{v} \cdot \mathbf{T},
$$




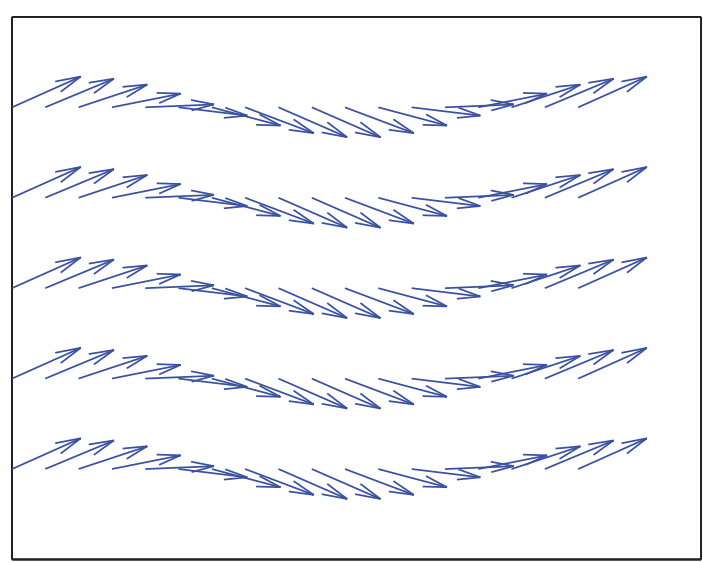

(a)

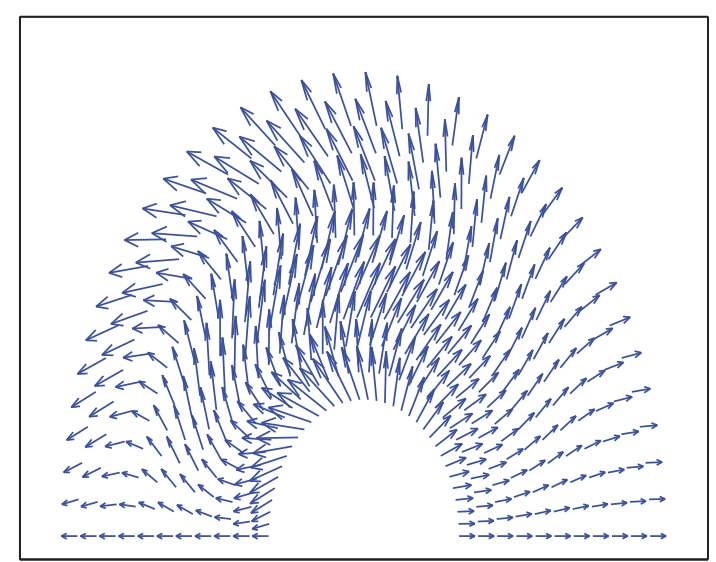

(b)

FIgURE 2: Time average of the acoustic Umov-Poynting vector. (a) Incident wave; (b) scattered wave.
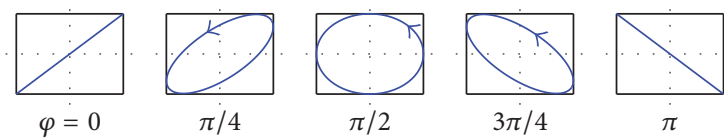

$\pi$

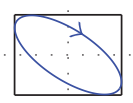

$5 \pi / 4$

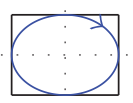

$3 \pi / 2$

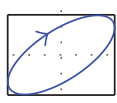

$7 \pi / 4$

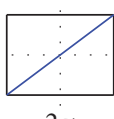

$2 \pi$

FIgURE 3: Paths of particle vibration in a spatial period.

where $\mathbf{v}$ is particle velocity and $\mathbf{T}$ is stress tensor. The acoustic Umov-Poynting vector characterizes the combined action of the particle velocity and the stress tensor. It represents the spatial instantaneous acoustic energy flux in solids.

\section{Particle Polarization and the Time-Space Average Method}

The acoustic energy flux vector is the instantaneous energy flux as a time-space function. The observation at different time or spatial locations differs. For example, acoustic energy flux vectors of the incident plane wave and the spherical scattered wave at time zero are shown in Figure 1.

In a fluid, the propagation of acoustic energy is generally described by a time average of the acoustic flux vector:

$$
\mathbf{I}=\frac{1}{T} \int_{0}^{T} \mathbf{P}(\mathbf{r}, t) \mathrm{d} t .
$$

Time average of acoustic Umov-Poynting vector is defined as the traditional acoustic intensity. It can accurately describe the propagation of acoustic energy in fluids. However, this method is no longer accurate in solids because of the spatial periodicity of the particle polarization. The time average of the acoustic Umov-Poynting vector for the incident plane wave and the spherical scattered wave over one spatial period are shown in Figure 2.

An obvious mistake could be found in Figure 2. When using traditional definition of acoustic intensity to describe the propagation of acoustic energy in solids, the energy propagation direction is not consistent with the wave propagation direction. Existing classical methods have the fault that they can not accurately show the wave propagation properties because the wave propagation direction is essentially the energy propagation direction.

The path of particle vibration in the acoustic field is elliptical because the compressional wave and the shear wave have different propagation velocities and the both have effect on particle velocity. A more accurate description method is needed according to the spatial periodicity of the polarization state of particle vibration. The paths of particle vibration in a spatial period are shown in Figure 3.

The spatial periodicity of the polarization state of particle vibration will inevitably result in the interference of acoustic energy in a direction perpendicular to the wave propagation direction. The acoustic energy flux wriggles surrounding the wave propagation direction.

Based on the foregoing analysis, a time-space average method of acoustic Umov-Poynting vector is proposed:

$$
\widehat{\mathbf{I}}=\frac{1}{L T} \int_{0}^{L} \int_{0}^{T} \mathbf{P}(\mathbf{r}, t) \mathrm{d} t \mathrm{~d} l,
$$

where $L=2 \pi /(K-k)$ is the distance of a spatial period in the wave propagation direction and $T$ is the time period of harmonic acoustic wave. This method is intended to eliminate the influence of the spatial period of the acoustic UmovPoynting vector. The time-space averages of the acoustic Umov-Poynting vector for the incident plane wave and the spherical scattered wave over one spatial period are shown in Figure 4.

Comparing Figure 2 with Figure 4, the time-space average method of acoustic Umov-Poynting vector could eliminate the influence of the interference of the local energy exchange and retain the trend of energy propagation. So this method is more accurate in describing the acoustic energy propagation 


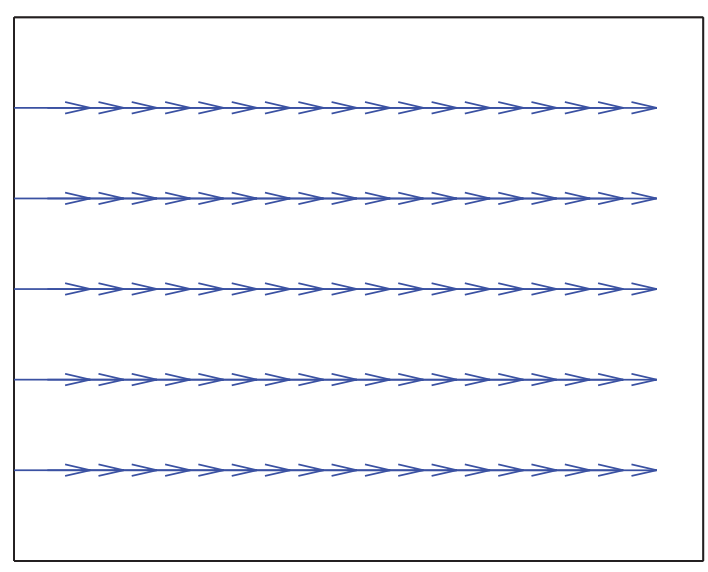

(a)

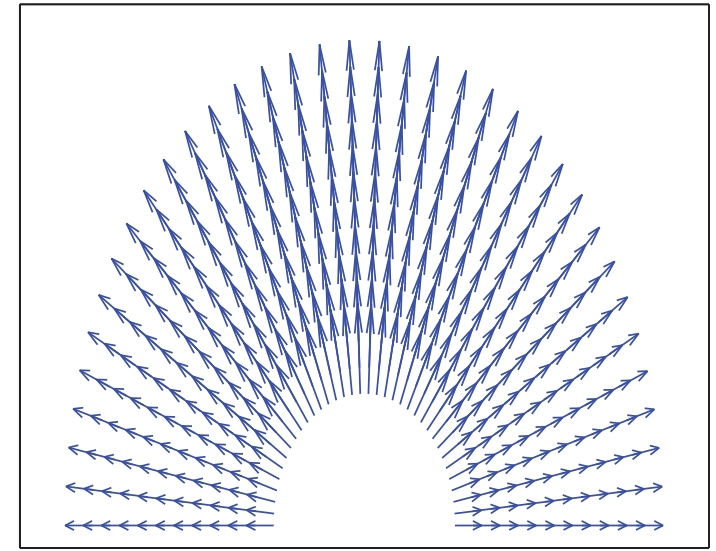

(b)

FIGURE 4: Time-space average of the acoustic Umov-Poynting vector. (a) Incident wave; (b) scattered wave.

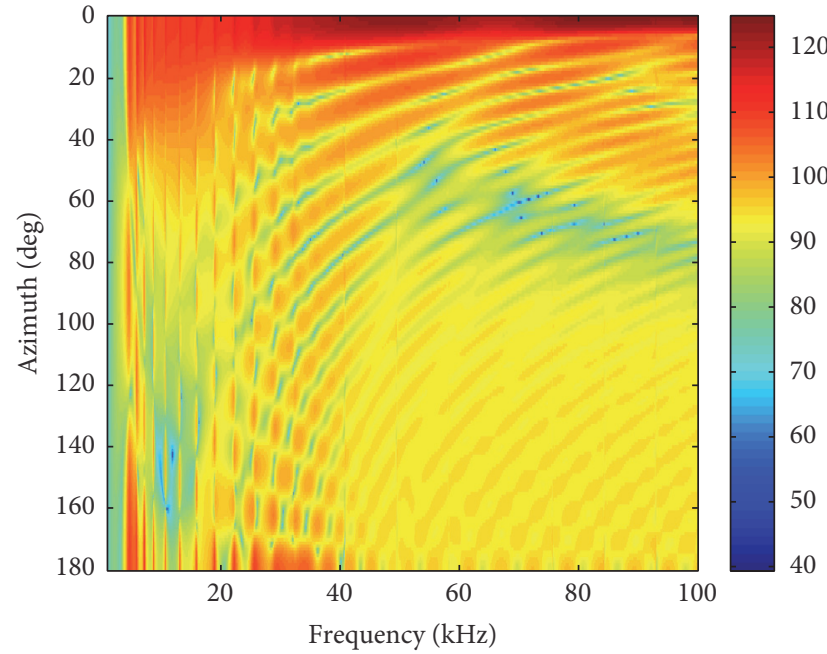

(a)

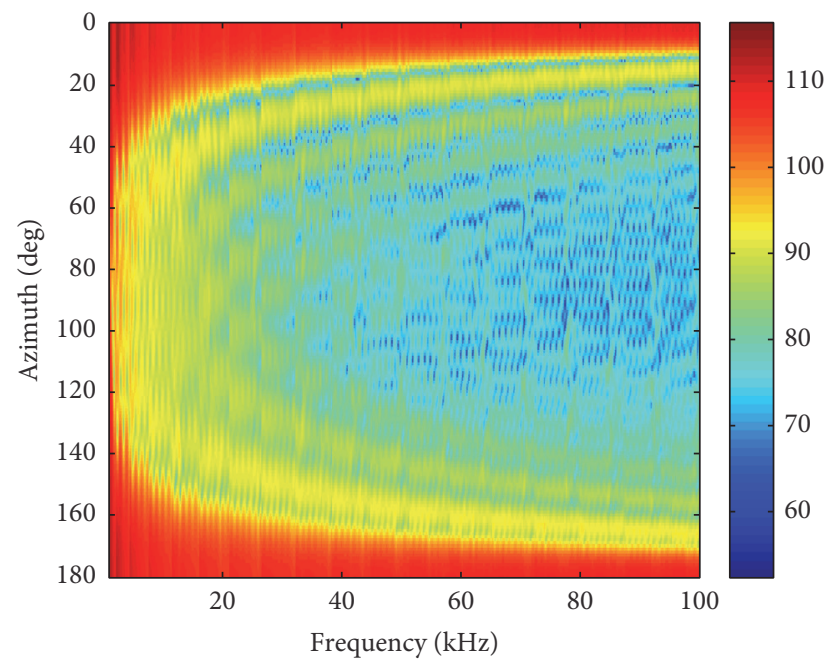

(c)

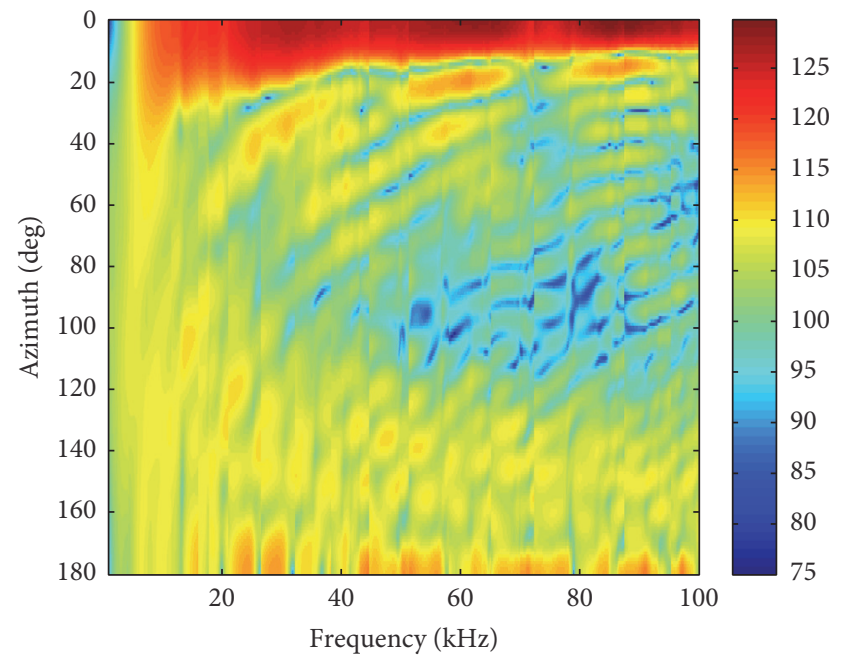

(b)

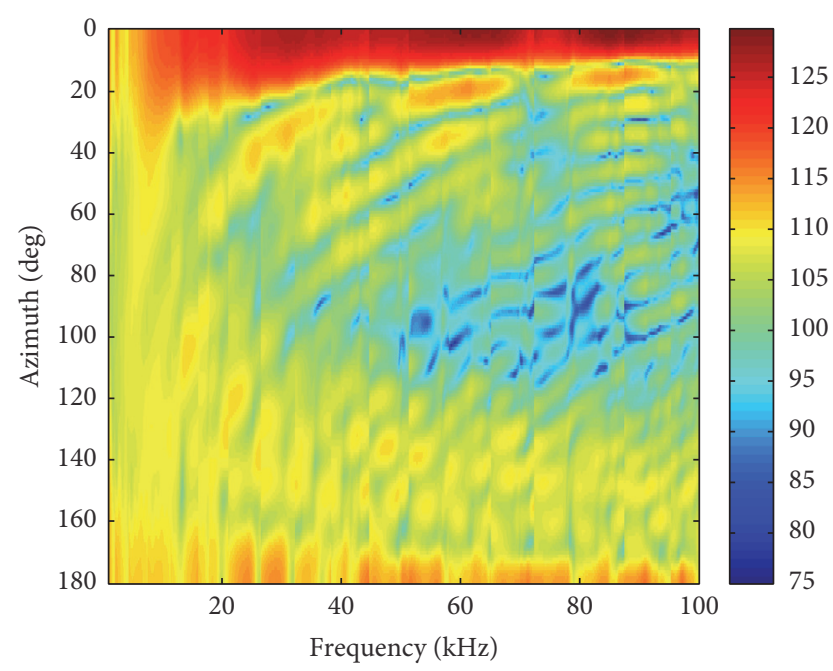

(d)

FIGURE 5: Calculation results of different models. (a) Model 1; (b) model 2; (c) model 3; (d) model 4. 
than the time average method. It clearly shows the properties of acoustic energy propagation in solids.

\section{Application on Scattering Properties of a Spherical Shell in Sediment}

4.1. Scattering Properties of a Spherical Shell in Sandy Sediment. The shear wave velocity in sandy sediment is much less than its compressional wave velocity $[16,17]$. In sandy sediment, the compressional wave velocity is $1700 \mathrm{~m} / \mathrm{s}$, whereas the shear wave velocity is $200 \mathrm{~m} / \mathrm{s}$ with a mass density of $1800 \mathrm{~kg} / \mathrm{m}^{3}$. The scatterer is a spherical steel shell. Its compressional wave velocity is $5792 \mathrm{~m} / \mathrm{s}$, the shear wave velocity is $3200 \mathrm{~m} / \mathrm{s}$, and the density is $7850 \mathrm{~kg} / \mathrm{m}^{3}$. The outer diameter of the spherical shell is $0.20 \mathrm{~m}$ and the inner diameter is $0.18 \mathrm{~m}$. The receiver distance from the spherical shell center is $100 \mathrm{~m}$.

Four kinds of scattering models are calculated in order to compare the differences among the different models. Model 1 is as follows: the incident wave is a compressional plane wave and the sediment is a fluid where the compressional wave velocity and the density are the same as in the sandy sediment. Model 2 is as follows: the incident wave is a compressional plane wave in a sandy sediment. Model 3 is as follows: the incident wave is a shear plane wave in a sandy sediment. Model 4 is as follows: the incident waves are a compressional and a shear plane wave in a sandy sediment. Azimuthfrequency color-maps of the scattering acoustic intensity level excited by a unit energy incident wave with a frequency range from $1 \mathrm{kHz}$ to $100 \mathrm{kHz}$ (the reference level is $10^{-12} \mathrm{~W} / \mathrm{m}^{2}$; the equivalent receiving distance is $1 \mathrm{~m}$ ) are shown in Figure 5.

The results in Figures 5(a) and 5(b) show a sharp difference in energy distribution between models 1 and 2 . The two models have the same compressional wave velocity, density, and incident wave type. But model 2 needs to consider the influence of the shear component of the scattered wave. Therefore, a sandy sediment can not be regarded as a fluid even if its shear wave velocity is much less than its compressional wave velocity.

The results in Figure 5(c) show that the scattered energy excited by the incident shear wave is different from that of the incident compressional wave. The scattering energy is mainly concentrated in the vicinity of the directions of backscattering and forward scattering. It will be more concentrated when the frequency of the incident wave gets higher. Furthermore, the scattered energy excited by the incident shear wave is obviously lower than that of incident compressional wave. Its contribution to the total scattered energy excited by the incident compressional and shear wave is significant only when the frequency is low. This property could be seen in both Figures 5(d) and 6.

4.2. Scattering Properties of a Spherical Shell in General Solid. The ratio of shear wave velocity and compressional wave velocity in solids is

$$
\frac{c_{T}}{c_{L}}=\sqrt{\frac{\mu}{\lambda+2 \mu}}=\sqrt{\frac{1-2 \sigma}{2-2 \sigma}} .
$$

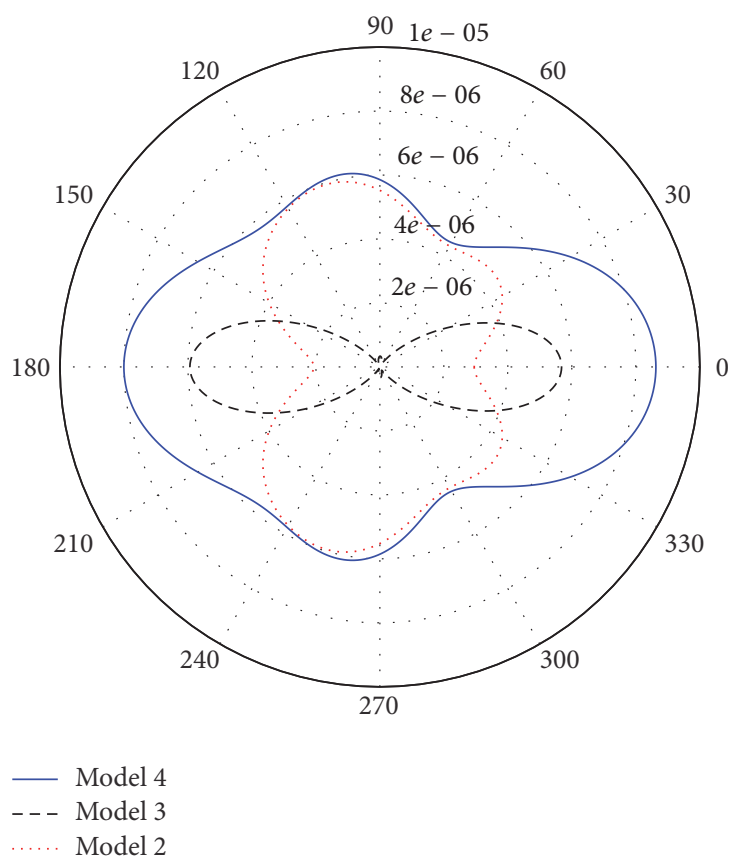

FIGURE 6: Energy contrast between models 2 and 4 .

Usually Poisson's ratio for a general solid is about 0.3 . That means that the ratio of shear wave velocity and compressional wave velocity in solid is about $1 / 2$. The case that the shear wave velocity is much less than the compressional wave velocity is infrequent. The shear wave velocity for sandy sediments can be set as a value of $850 \mathrm{~m} / \mathrm{s}$ while other conditions remain unchanged. The last three models are calculated again and the results are shown in Figure 7.

The results in Figure 7 show that the scattering properties of a general solid are different from that of a sandy sediment. The results of the three models are influenced by the change in shear wave velocity. The scattered energy excited by an incident shear wave is mainly concentrated in the vicinity of the forward scattering. The backscattering energy fluctuated with the frequency of the incident wave. The influence on the total scattered energy is also highly significant.

\section{Conclusions}

In solids, the polarization state of particle vibration is a spatially periodic function in the wave propagation direction. It is difficult to establish the connection between the energy propagation direction and the physical quantities of the acoustic field. According to the polarization state of the particle vibration and the energy propagation direction, a time-space averaging method based on the acoustic energy flux is proposed. The method could eliminate the influence of the interference of the local energy exchange and retain the trend of the energy propagation.

Several conclusions are illustrated through the analysis properties of the scattered energy distribution for a steel shell in sandy sediment and for a general solid.

(1) Although the shear wave velocity for a sandy sediment is much less than its compressional wave velocity, 


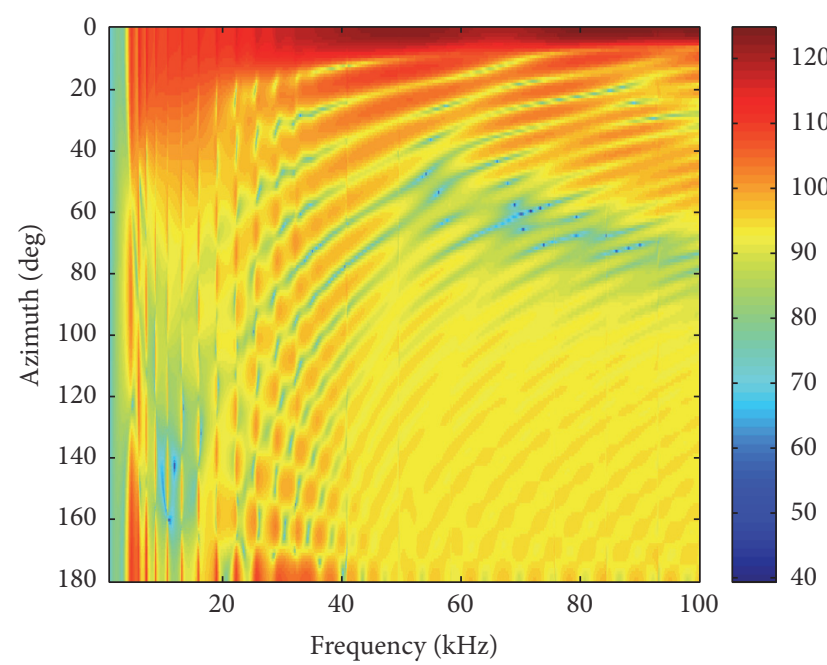

(a)

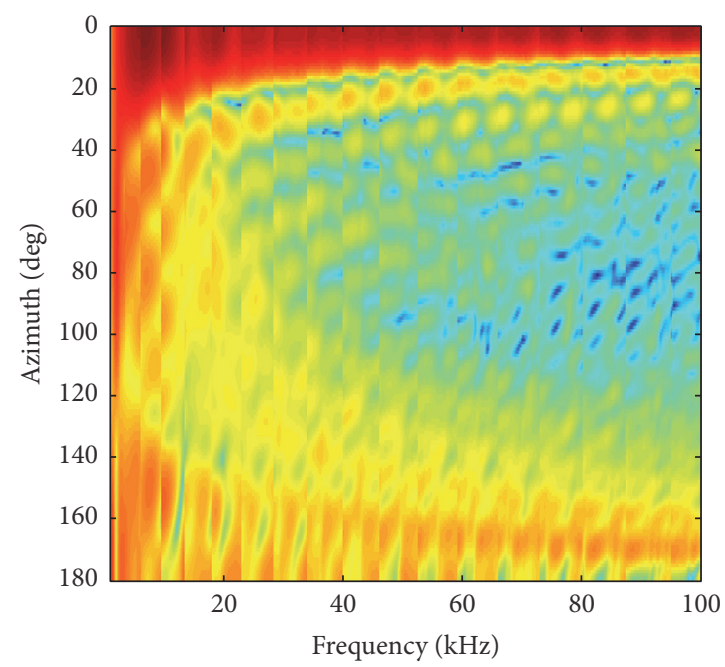

(c)

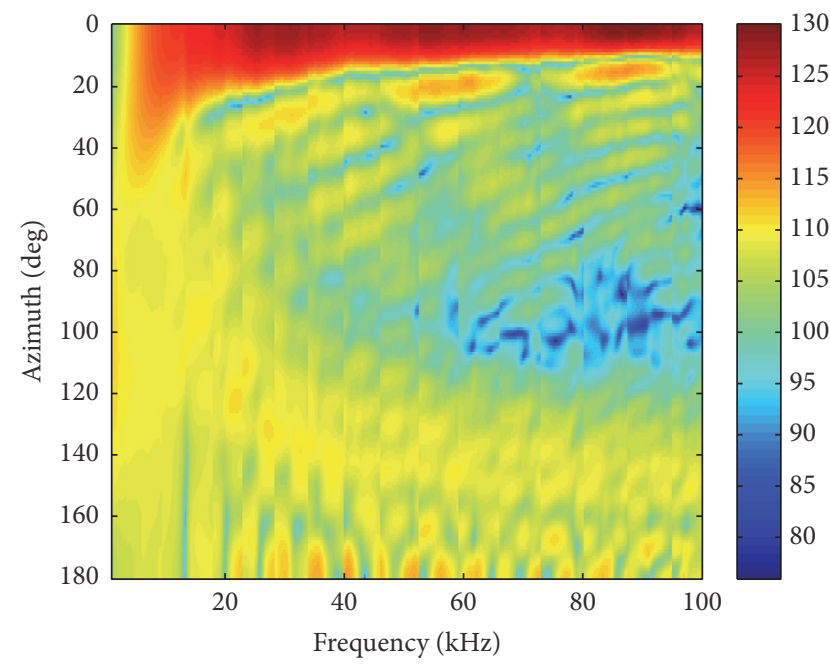

(b)

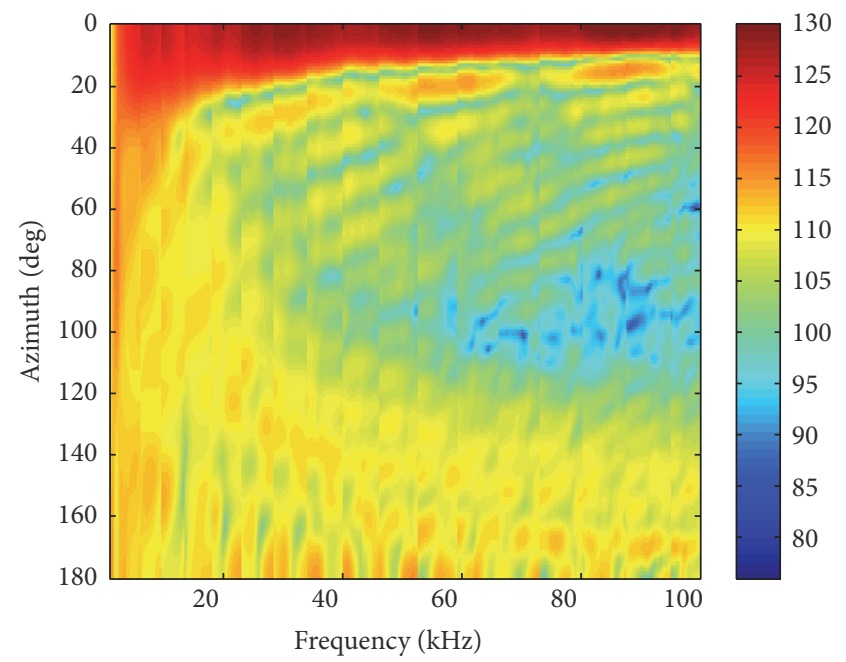

(d)

FIGURE 7: Calculation results of different models. (a) Model 1; (b) model 2; (c) model 3; (d) model 4.

sandy sediment can not be regarded as a fluid nor a general solid. The results show that its scattering properties are different from a fluid and a general solid.

(2) In sandy sediments, the scattered energy excited by a shear wave incident mainly concentrates in the vicinity of the direction of backscattering and forward scattering, whereas, in a general solid, the scattered energy mainly concentrates in the vicinity of the direction of forward scattering, no matter if it is excited by an incident compressional wave or a shear wave.

(3) The scattered energy excited by an incident shear wave plays an important role in the total scattered energy excited by an incident compressional wave and a shear wave at low frequency.

\section{Competing Interests}

The authors declare that there is no conflict of interests regarding the publication of this paper.

\section{Acknowledgments}

This study is supported by the Opening Foundation of National Laboratory of Underwater Acoustic Technology (no. 2016001).

\section{References}

[1] C. F. Ying and R. Truell, "Scattering of a plane longitudinal wave by a spherical obstacle in an isotropically elastic solid," Journal of Applied Physics, vol. 27, no. 9, pp. 1086-1097, 1956.

[2] N. G. Einspruch, E. J. Witterholt, and R. Truell, "Scattering of a plane transverse wave by a spherical obstacle in an elastic 
medium," Journal of Applied Physics, vol. 31, no. 5, pp. 806-818, 1960.

[3] N. G. Einspruch and R. Truell, "Scattering of a plane longitudinal wave by a spherical fluid obstacle in an elastic medium," Journal of the Acoustical Society of America, vol. 32, no. 2, pp. 214-220, 1960.

[4] A. D. Pierce, "Energy flux associated with fundamental elastic wave types on inhomogeneous cylindrical shells," The Journal of the Acoustical Society of America, vol. 85, no. 117, 1989.

[5] J. M. Carcione and F. Cavallini, "Energy balance and fundamental relations in anisotropic-viscoelastic media," Wave Motion, vol. 18, no. 1, pp. 11-20, 1993.

[6] J. M. Carcione, "Reflection and transmission of $\mathrm{qP}-\mathrm{qS}$ plane waves at a plane boundary between viscoelastic transversely isotropic media," Geophysical Journal International, vol. 129, no. 3, pp. 669-680, 1997.

[7] M. A. Ainslie and P. W. Burns, "Energy-conserving reflection and transmission coefficients for a solid-solid boundary," Journal of the Acoustical Society of America, vol. 98, no. 5, pp. 28362840, 1995.

[8] V. V. Kotlyar, A. G. Nalimov, and R. V. Skidanov, "Calculation of Umov-Poynting vector and the electromagnetic wave pressure force on a homogeneous dielectric cylinder," in Proceedings of the Saratov Fall Meeting, vol. 5773, pp. 106-118, 2004.

[9] M. L. Rumerman, "Radiated power with varying restraints against rotation and translation," Shock and Vibration, vol. 14, pp. 81-88, 2007.

[10] C. E. Dean and J. P. Braselton, "Visualization of the energy flow and around a fluid-loaded elastic cylindrical shell," Journal of the Acoustical Society of America, vol. 107, no. 5, pp. 2921-2921, 2000.

[11] C. E. Dean and J. P. Braselton, "An improved method of visualizing the energy flow: the Rayleigh wave," Journal of the Acoustical Society of America, vol. 123, no. 5, p. 3312, 2008.

[12] C. E. Dean and J. P. Braselton, "Visualization of the energy flux in an ensonified fluid-loaded elastic sphere," The Journal of the Acoustical Society of America, vol. 103, no. 5, pp. 119-125, 1998.

[13] N. G. Kuts, "Application of the Umov-Poynting vector for solving heat transfer problems in technology," Scientific Technical Review, vol. 63, no. 4, pp. 10-16, 2013.

[14] S. A. Nazarov, "The Mandelstam energy radiation conditions and the Umov-Poynting vector in elastic waveguides," Journal of Mathematical Sciences, vol. 195, no. 5, pp. 676-729, 2013.

[15] G. Wang, W.-Y. Li, W. L. Li, and J. Du, "Sound radiation from an elastically restrained plate covered by an acoustic decoupling layer," Mathematical Problems in Engineering, vol. 2015, Article ID 659472, 11 pages, 2015.

[16] Z. Y. Zhang and C. T. Tindle, "Improved equivalent fluid approximations for a low shear speed ocean bottom," Journal of the Acoustical Society of America, vol. 98, no. 6, pp. 3391-3396, 1995.

[17] M. Kimura, "Shear wave speed dispersion and attenuation in granular marine sediments," Journal of the Acoustical Society of America, vol. 134, no. 1, pp. 144-155, 2013. 


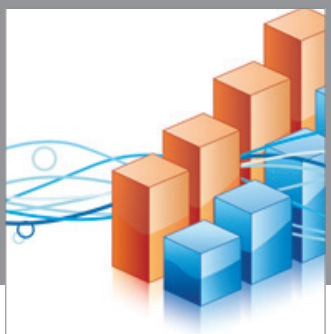

Advances in

Operations Research

vatem alat4

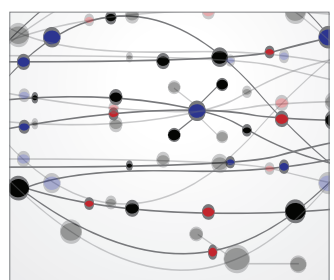

\section{The Scientific} World Journal
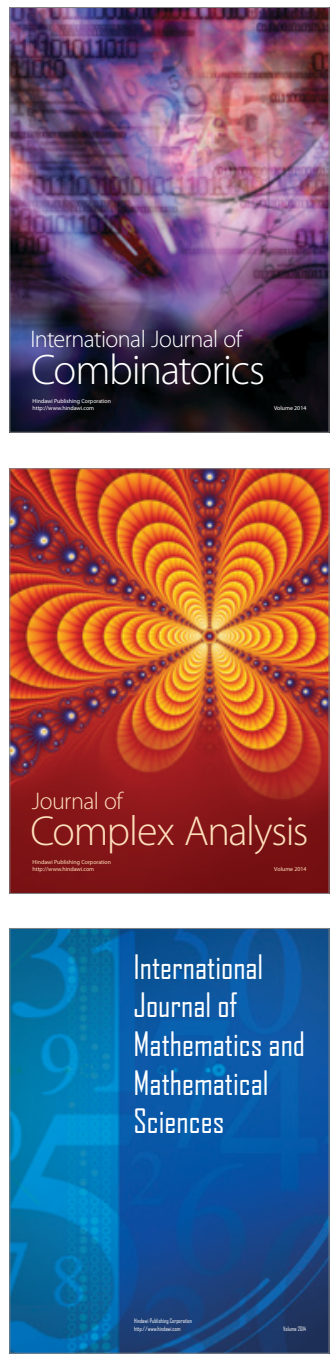
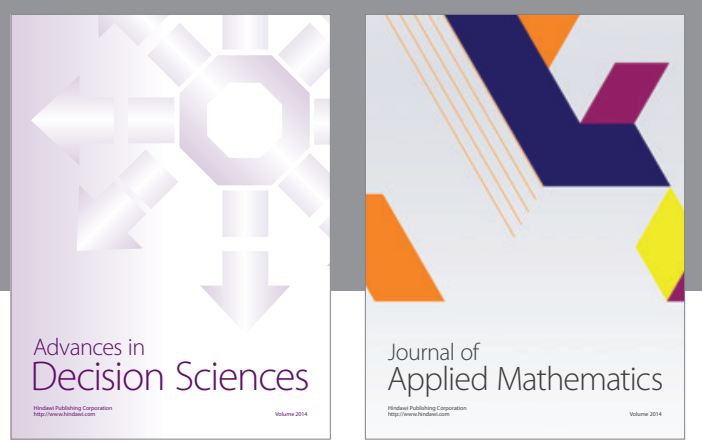

Algebra

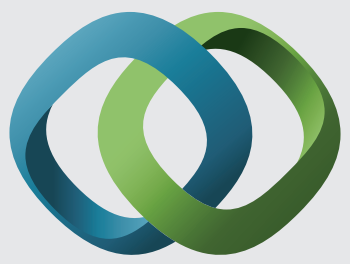

\section{Hindawi}

Submit your manuscripts at

https://www.hindawi.com
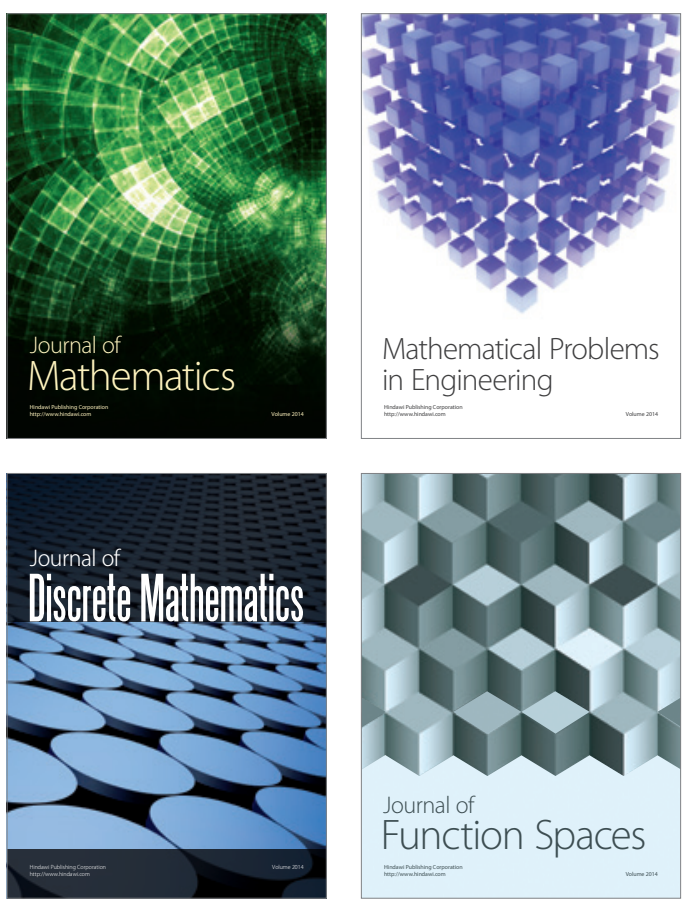

Mathematical Problems in Engineering
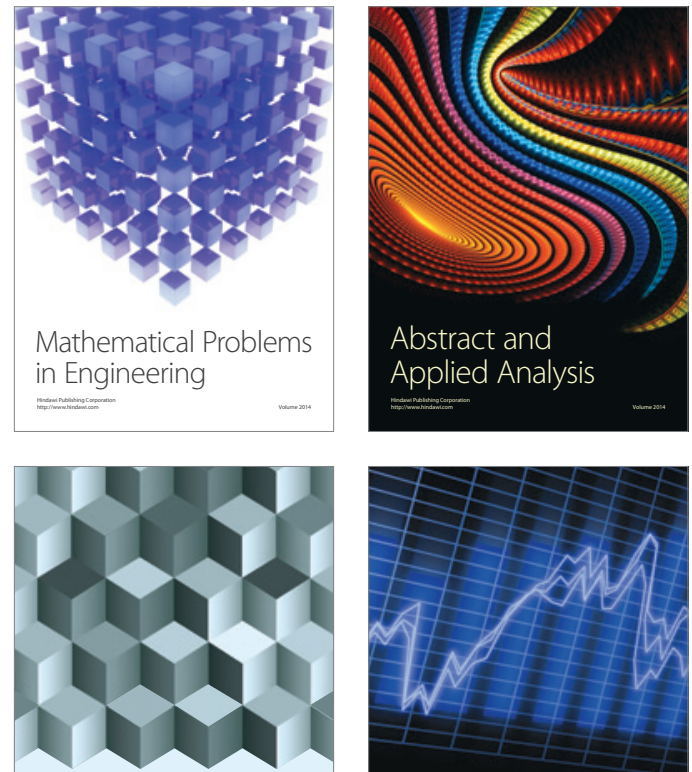

Journal of

Function Spaces

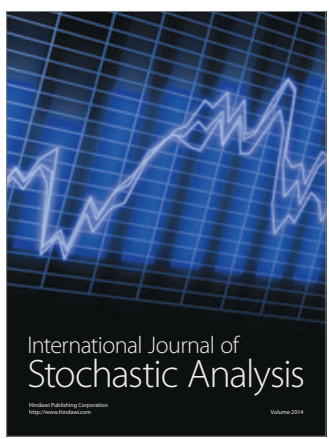

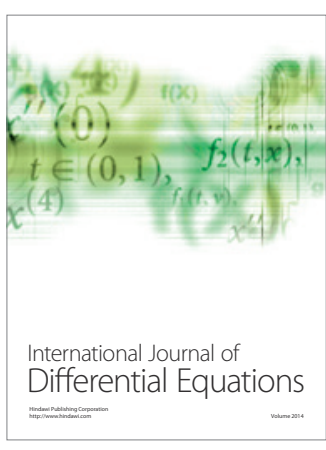
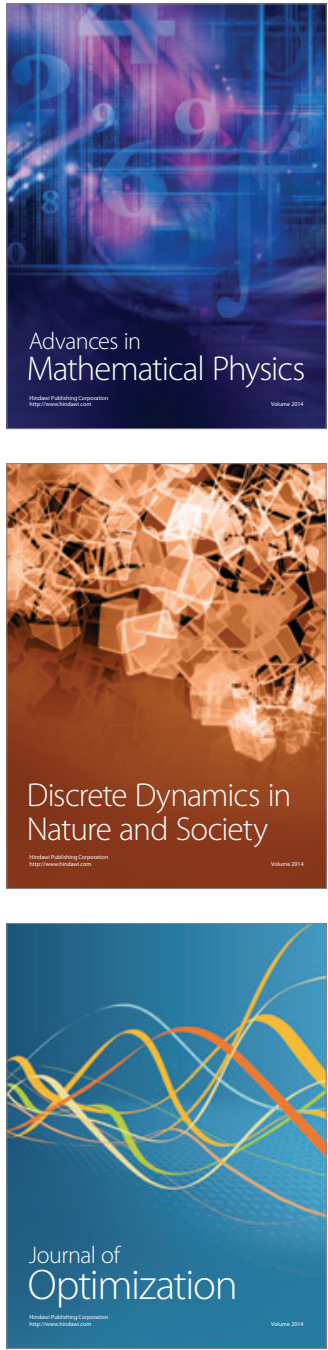